\title{
El triaje: herramienta fundamental en urgencias y emergencias
}

\section{Triage: a key tool in emergency care}

\author{
W. Soler ${ }^{3}$, M. Gómez Muñoz ${ }^{3}$, E. Bragulat ${ }^{2}$, A. Álvarez ${ }^{1}$
}

\section{RESUMEN}

El triaje es un proceso que permite una gestión del riesgo clínico para poder manejar adecuadamente y con seguridad los flujos de pacientes cuando la demanda y las necesidades clínicas superan a los recursos. Actualmente se utilizan sistemas de triaje estructurado con cinco niveles de prioridad que se asignan asumiendo el concepto de que lo urgente no siempre es grave y lo grave no es siempre urgente y hacen posible clasificar a los pacientes a partir del "grado de urgencia", de tal modo que los pacientes más urgentes serán asistidos primero y el resto serán reevaluados hasta ser vistos por el médico. El sistema español de triaje (SET) y el sistema de triaje Manchester (MTS) son los dos sistemas normalizados de mayor implantación en nuestro país, pero analizamos también el sistema de triaje elaborado en Navarra que se integra en la historia clínica informatizada ( $\mathrm{HCI})$ y que se utiliza en toda la red pública hospitalaria de Navarra. Todos son sistemas multidisciplinares basados en motivos y en la urgencia de la consulta pero no en diagnósticos y son llevados a cabo por enfermería con apoyo médico puntual. También todos incorporan elementos de monitorización de la calidad del propio servicio de urgencias y cuentan con proyección para poder ser aplicados en el ámbito extrahospitalario.

Palabras clave. Triaje. Servicio de Urgencia Hospitalario. Indicadores de calidad. Sistema de Triaje Manchester. Sistema de triaje español.

\begin{abstract}
"Triage" is a process that enables us to manage clinical risk in order to safely and suitably handle patient flows when demand and clinical needs exceed resources. At present, triage systems that are employed are structured according to five levels of priority. Levels are allocated according to the concept that what is urgent is not always serious and that what is serious is not always urgent. This makes it possible to classify patients according to "degree of urgency", so that the more urgent patients will be attended to first and the rest will be re-evaluated until they are seen by the doctor. The Spanish triage system (SET) and the Manchester triage system (MTS) are the two standardised systems most implemented in our country. We also discuss the system of triage devised in Navarre - integrated in the computerised clinical history- and used in the hospital network of Navarre. All are multidisciplinary systems based on the reasons and urgency of consultation, but not on diagnoses, and are carried out by nursing staff with medical support when required. In addition, they all include monitoring of the quality of the accident and emergency service itself, and can be applied in the outpatient field.
\end{abstract}

Key words. Triage. Hospital Urgency and Emergency Service. Quality indicators. Manchester Triage System. Spanish Triage System.
1. Servicio de Urgencias. Hospital Central de Asturias.

2. Servicio de Urgencias. Hospital Clínic. Barcelona.

3. Servicio de Urgencias. Hospital Virgen del Camino. Pamplona.

\author{
Correspondencia \\ Wifredo Soler Pérez \\ Jefe del Servicio Urgencias \\ Hospital Virgen del Camino \\ Irunlarrea, 4 \\ 31008 Pamplona \\ Email: wsolerpe@navarra.es
}




\section{INTRODUCCIÓN}

El término triage o triaje es un neologismo que proviene de la palabra francesa trier que se define como escoger, separar o clasificar. Desde que este término comenzó a utilizarse en las batallas napoleónicas persiste como concepto de clasificación o priorización de la atención urgente de pacientes.

Los servicios de urgencias en España vienen padeciendo un aumento permanente de la demanda, de tal forma que hemos pasado de los 18 millones de urgencias en 1977 a 26,2 en $2007^{1}$, hecho que dificulta la atención rápida y eficaz que se les exige. Esto es debido, al menos en parte, a la elevada utilización de los servicios de urgencias para la atención de situaciones no urgentes, según estudios hasta el $70 \% 2$, que pueden condicionar demoras en la asistencia de pacientes graves además de generar consecuencias negativas para el conjunto del hospital, incluyendo el incremento de costes asistenciales ${ }^{3}$.

Paralelamente, en esta década se ha asistido a una transformación profunda de los servicios de urgencias hospitalarios (SUH) con cambios estructurales, organizativos y funcionales para adaptarse a esta situación ${ }^{4}$, siendo el sistema de triaje hospitalario uno de los puntos clave en dichas modificaciones.

\section{DEFINICIÓN}

El «triaje/clasificacion» es un proceso que nos permite una gestión del riesgo clínico para poder manejar adecuadamente $y$ con seguridad los flujos de pacientes cuando la demanda y las necesidades clínicas superan a los recursos. Debe ser la llave de entrada a una asistencia eficaz y eficiente, y por tanto, una herramienta rápida, fácil de aplicar y que además poseen un fuerte valor predictivo de gravedad, de evolución y de utilización de recursos ${ }^{5}$.

La aplicación del triaje, tal como lo conocemos, proviene del mundo anglosajón que desarrolla a partir de los años 60 diversos planteamientos basados en escalas de 3 ó 4 niveles de categorización ${ }^{6}$ que, al no haber demostrado suficiente fiabilidad, relevancia y valide ${ }^{7}$, han sido casi todas sustituidas a partir de los años 90 por nuevas escalas de 5 niveles de priorización que pueden ser aplicadas en modelos del denominado triaje estructurado ${ }^{8}$.

La aplicación de dichas escalas parte de un concepto básico en triaje: lo urgente no siempre es grave y lo grave no es siempre urgente $^{9}$. Ello hace posible clasificar a los pacientes a partir del "grado de urgencia", de tal modo que los pacientes más urgentes serán asistidos primero y el resto serán reevaluados hasta ser vistos por el médico. Se acepta, en consecuencia, que las funciones del triaje deben ser $^{10}$ :

1. Identificación de pacientes en situación de riesgo vital.

2. Asegurar la priorización en función del nivel de clasificación.

3. Asegurar la reevaluación de los pacientes que deben esperar.

4. Decidir el área más apropiada para atender a los pacientes.

5. Aportar información sobre el proceso asistencial.

6. Disponer de información para familiares.

7. Mejorar el flujo de pacientes y la congestión del servicio.

8. Aportar información de mejora para el funcionamiento del servicio.

Actualmente se reconocen cinco modelos de triaje estructurado con una amplia implantación:

1. La Australian Triage Scale (ATS)

2. La Canadian Emergency Department Triage and Acuity Scale (CTAS)

3. El Manchester Triage System (MTS)

4. El Emergency Severit Index (ESI)

5. El Sistema Español de Triage (SET) adoptado por la Sociedad Española de Medicina de Emergencias (SEMES) a partir del Model Andorrá de Triatge: MAT

A nivel del estado español, la $\mathrm{MTS}^{11}$ y el $\mathrm{SET}^{12}$ son los dos sistemas que han alcanza- 
do mayor difusión, aunque también coexisten con otros de implantación local, más adaptados a su propia realidad, como pueden ser el "Sistema Donostiarra de Triaje» del Hospital Donostia, el Sistema de Triaje de la red pública de hospitales de Navarra, estos dos sobre escalas de cinco prioridades, o el sistema de clasificación en base a escalas de cuatro niveles de priorización (Plan Andaluz de urgencias y Emergencias de la Consejería de Salud).

\section{TRIAJE COMO INDICADOR Y CONTROL DE CALIDAD}

La disponibilidad de un sistema de triaje estructurado en los SHU ha sido considerado como un índice de calidad básico y relevante de la relación riesgo-eficiencia aceptándose que el porcentaje de pacientes dentro de cada nivel de triaje es como la "huella digital» del SUH, de tal forma que se puede establecer cuál es el perfil del SHU en función al nivel de urgencia y la intensidad del servicio que se presta ${ }^{13}$.

Gómez Jiménez propone cuatro índices de calidad ${ }^{10}$ que deben ser asumidos por el sistema de triaje implantado y que se convierten en testigos de su capacidad como indicador de calidad del propio servicio de urgencias. Son:

1. El índice de pacientes perdidos sin ser vistos por el médico ( $\leq$ del $2 \%$ de todos los pacientes que acuden a urgencias). Divide a este índice en aquéllos que se pierden sin ser clasificados y aquéllos que se trian pero se van antes de ser visitados.

2. Tiempo desde la llegada a urgencias hasta que se inicia la clasificación (menor de 10 minutos).

3. Tiempo que dura la clasificación (menor de cinco minutos como recomendación)

4. Tiempo de espera para ser visitado, establecido en cada uno de los niveles de prioridad de que conste el sistema de triaje y que varía entre la atención inmediata del nivel I de prioridad hasta los 240 minutos, con- siderados como el tiempo máximo que debe esperar la prioridad menos urgente $^{11,8,14-16}$.

En cuanto a este último indicador de calidad, se establecen niveles de priorización en la atención. Cada nivel va a determinar el tiempo óptimo entre la llegada y la atención y cada modelo de triaje estructurado establece cuáles son esos tiempos ideales, que varían muy poco de un modelo a otro. Todas las escalas comentadas anteriormente coinciden ampliamente en estos parámetros:

- Nivel I: prioridad absoluta con atención inmediata y sin demora.

- Nivel II: situaciones muy urgentes de riesgo vital, inestabilidad o dolor muy intenso. Demora de asistencia médica hasta 15 minutos.

- Nivel III: urgente pero estable hemodinámicamente con potencial riesgo vital que probablemente exige pruebas diagnósticas y/o terapéuticas. Demora máxima de 60 minutos.

- Nivel IV: urgencia menor, potencialmente sin riesgo vital para el paciente. Demora máxima de 120 minutos.

- Nivel V: no urgencia. Poca complejidad en la patología o cuestiones administrativas, citaciones, etc. Demora de hasta 240 minutos.

Estos cinco niveles se establecen en base a:

- Descriptores clínicos, síntomas centinela o categorías sintomáticas, abiertas o cerradas, con o sin ayuda de algoritmos o diagramas.

- Discriminantes del nivel de urgencia: riesgo vital, constantes fisiológicas, tiempo de evolución, nivel de dolor, mecanismo de lesión, etc.

Un sistema estructurado de triaje puede servir también para valorar otra serie de parámetros, como son el tiempo de estancia en urgencias, el porcentaje de ingresos en función del nivel de gravedad, las necesidades de recursos, etc. que indirectamente van a relacionarse con cada uno de los niveles de prioridad ${ }^{17}$ y que están estrechamente condicionados por el incremento 
de la demanda, la cual depende tanto de determinantes externos (afluencia) como internos (propia capacidad del servicio, demora de exploraciones, espera de cama para ingreso, espera de transporte, problemas sociosanitarios ${ }^{9}$ ). Estos parámetros son fundamentales para orientar la gestión organizativa, económica y el funcionamiento del propio servicio de urgencias ${ }^{16,18}$.

\section{¿QUIÉN LLEVA A CABO EL TRIAJE?}

Dado que el triaje no se fundamenta en diagnósticos y la concordancia interobservador hallada en las diferentes escalas ha resultado muy satisfactoria ${ }^{19}$, el triaje es reivindicado desde sus inicios por enfermería $^{20}$, que consigue muy buenos resultados incluso con escalas de 4 niveles de prioridad $^{21}$. Hay, sin embargo, sistemas que defienden que debe ser un médico experimentado de urgencias el encargado de realizarlo (sistema donostiarra de triaje).

Actualmente es el personal de enfermería el que con más frecuencia lo realiza ${ }^{22}$, con o sin ayuda del facultativo. En este sentido algunos estudios concluyen que el triaje de enfermería con apoyo médico es más eficiente que el triaje de enfermería aislado ${ }^{23,24}$ sobre todo para los casos de alta urgencia y complejidad ${ }^{25}$. No obstante, otros modelos definen el "triaje avanzado", en el que el profesional de enfermería ejecuta un procedimiento o administra una medicación en base a directrices médicas con protocolos, circuitos y órdenes médicas preestablecidos. Estos protocolos se han de poner en práctica una vez consensuados y establecidos y después de que la mayoría de los profesionales de enfermería hayan recibido la formación adecuada y se haya verificado su capacitación. También deben ser aprobados por la estructura hospitalaria competente ${ }^{5}$.

\section{TRIAJE PEDIÁTRICO}

Todas las escalas que hemos nombrado se han diseñado para ser aplicadas a la población adulta y secundariamente se han desarrollado adaptaciones dirigidas a la población pediátrica que no siempre han demostrado la misma validez por las diferencias clínicas entre ambas poblaciones. Así, pues se ha objetivado que el triaje pediátrico presenta unas características propias $^{26}$.

- Menor grado de urgencia (mayor porcentaje de niveles IV y V).

- El nivel I se concentra en los niños de menor edad.

- Menor complejidad para un mismo nivel de urgencia.

- Menor tiempo de estancia, de consumo de recursos e índice de ingreso para un mismo nivel de triaje que en adultos.

- Preponderancia de los procesos infecciosos y febriles.

- Las 10 categorías sintomáticas más frecuentes en los niños aglutinan a casi la totalidad de los motivos de consulta lo que facilita el que se pueda conseguir la formación específica en triaje pedriátrico que necesitan los profesionales. Esto facilita el objetivo de la especial formación que se debe impartir a los profesionales.

\section{MODELOS DE SISTEMA DE TRIAJE}

Se describe de manera esquemática las características de los dos sistemas de triaje con mayor implantación en España, el SET y el MTS y, a continuación, el sistema que se ha elaborado para la red pública de hospitales de Navarra adaptado a partir de los dos primeros.

\section{SISTEMA ESPAÑOL DE TRIAJE (SET)}

Gómez Jiménez y colaboradores desarrollan en junio de 2000, en el Hospital Nostra Senyora de Meritxell de Andorra, un nuevo sistema de triaje estructurado denominado "Model Andorrá de Triatje» (MAT), que nace de una adaptación conceptual de la CTAS y convierte una escala basada en síntomas y diagnósticos centinela, en una 
escala basada en categorías sintomáticas con discriminantes clave y con algoritmos clínicos en formato electrónico. Sus principios fundamentales son ${ }^{27}$ :

- Triaje de 5 niveles normalizado, con un programa informático de gestión del triaje y otro de ayuda a la decisión clínica en el triaje (PAT).

- Modelo de triaje de enfermería no excluyente, que prioriza la urgencia del paciente sobre cualquier otro planteamiento.

- Integrado en un sistema de mejoría continua de la calidad, con seguimiento de indicadores de calidad en el triaje.
- Debe integrarse en un modelo global de historia clínica electrónica.

En 2003 fue asumido por la Sociedad Española de Medicina de Urgencias y Emergencias (SEMES) ${ }^{27}$ como el modelo estándar de triaje en castellano para todo el territorio español y se denominó «Sistema Español de Triaje» (SET).

En la tabla 1 se muestran las asociaciones previstas entre escalas, nivel de gravedad y tiempo de atención y en la tabla 2 el percentil de cumplimiento marginal que incluye los pacientes que han quedado fuera del primer tiempo de atención y que marca el SET y que deben ser visitados en un tiempo determinado para cada nivel de triaje $^{28}$.

Tabla 1. Relación entre escalas y niveles de gravedad en el SET.

\begin{tabular}{|c|l|l|l|}
\hline Nivel & \multicolumn{1}{|c|}{ Color } & \multicolumn{1}{c|}{ Categoría } & \multicolumn{1}{|c|}{ Tiempo de atención } \\
\hline I & Azul & Reanimación & Inmediato \\
\hline II & Rojo & Emergencia & $\begin{array}{l}\text { Inmediato enfermería/ } \\
\text { Médicos } 7 \text { minutos }\end{array}$ \\
\hline III & Naranja & Urgente & 30 minutos \\
\hline IV & Verde & Menos urgente & 45 minutos \\
\hline V & Negro & No urgente & 60 minutos \\
\hline
\end{tabular}

SET: Sistema español de triaje

Tabla 2. Percentil de cumplimiento y el percentil de cumplimiento marginal.

\begin{tabular}{|c|c|c|}
\hline Nivel & $\begin{array}{c}\text { Percentil cumplimiento } \\
\text { Percentil cumplimento marginal }\end{array}$ & $\begin{array}{c}\text { Tiempo de atención } \\
\text { Tiempo de atención }\end{array}$ \\
\hline I & $100 \%$ & 7 minutos \\
\hline II & $95 \%$ & inmediato enfermería / médicos 15 minutos \\
\hline II & $100 \%$ & 7 minutos enfermería / médicos 20 minutos \\
\hline III & $85 \%$ & 30 minutos \\
\hline III & $90 \%$ & 45 minutos \\
\hline III & $100 \%$ & 60 minutos \\
\hline IV & $85 \%$ & 120 minutos \\
\hline IV & $100 \%$ & 120 minutos \\
\hline V & $80 \%$ & 240 minutos \\
\hline V & $100 \%$ & \\
\hline
\end{tabular}




\section{Categorías sintomáticas}

Son un conjunto de síntomas o síndromes que el profesional interpreta y reconoce a partir del motivo de consulta referido por el paciente y que permiten clasificarlo dentro de una misma categoría clínica.

EI SET reconoce 32 categorías sintomáticas y 14 subcategorias que agrupan 578 motivos clínicos de consulta, todos vinculados a las diferentes categorías y subcategorías sintomáticas.

\section{Discriminantes y escalas de gravedad}

Un discriminante es un factor que permite diferenciar el grado de urgencia entre niveles de triaje. El SET utiliza:

- Constantes: discriminan a los pacientes con la misma sintomatología, entre el nivel II y el III (temperatura, presión arterial sistólica, frecuencia cardiaca, SpO2, glucemia capilar, Glasgow, NIHSS).

- Signos vitales anormales (estado de la piel, estado del pulso radial, frecuencia y profundidad respiratoria y somnolencia o confusión).

- Dolor: el SET dispone de una guía clínica de evaluación el dolor en el triaje que permite objetivar los valores que los pacientes manifiestan.

\section{Escalas de gravedad}

Las escalas de Glasgow, del coma no traumático, de grados de deshidratación, de gravedad clínica de la disnea o de gravedad clínica del asma entre otras, están disponibles para el profesional mediante el programa de ayuda PAT.

\section{Abordaje de la urgencia}

En el SET la diferenciación entre niveles se lleva a cabo mediante discriminantes, preguntas específicas y la aplicación de escalas de gravedad.

La escala de triaje del SET se estructura en base a una serie de escalas genera- les que se van incluyendo en las diferentes categorías sintomáticas. A partir de cada categoría sintomática se desarrollan los algoritmos que integran la escala de triaje y aspectos específicos relacionados con los motivos de consulta incluidos en la categoría sintomática.

Las categorías sintomáticas del SET tienen su desarrollo algorítmico en el Programa de Ayuda al Triaje (web e-PAT), de manera que cualquier entrada sintomática acabará rindiendo un nivel de triaje, una vez contestadas las preguntas planteadas por el programa.

\section{Calidad y monitorización}

Es un aspecto importante en el SET. Contempla, por un lado, indicadores de calidad y, por otro, el conjunto mínimo de datos asistenciales de urgencias (CMBDU) para realizar análisis de comparación con estándares o benchmarking, tanto en adultos como en niños y todo ello relacionado con los niveles de triaje.

El CMBDU hace posible también el análisis de casuística o case-mix de los pacientes atendidos en urgencias que se realiza principalmente en base a dos parámetros:

- Grado de urgencia: nivel de triaje asignado

- Complejidad: medida al clasificar a los pacientes en grupos según su destino desde urgencias (GRUDs) y según la edad (GRUDEs).

\section{SISTEMA MANCHESTER (MTS)}

El Manchester Triage Group nació en el mes de noviembre de 1994 y sus esfuerzos se centraron en 5 objetivos ${ }^{29}$ :

1. Elaborar una nomenclatura común.

2. Usar definiciones comunes.

3. Desarrollar una metodología sólida de triaje.

4. Implantar un modelo global de formación.

5. Permitir y facilitar la auditoria del método de triaje desarrollado. 
Se estableció un sistema de clasificación de 5 niveles y a cada una de estas nuevas categorías o niveles se le atribuyó un número, un color y un nombre que se defi- nió en términos de "tiempo clave» $\mathrm{o}$ "tiempo máximo para el primer contacto con el terapeuta ${ }^{29}$ (Tabla 3).

Tabla 3. Niveles de clasificación de la MTS.

\begin{tabular}{|c|l|c|c|}
\hline Número & Nombre & Color & Tiempo máximo \\
\hline 1 & Atención inmediata & Rojo & 0 \\
\hline 2 & Muy urgente & Naranja & 10 \\
\hline 3 & Urgente & Amarillo & 60 \\
\hline 4 & Normal & Verde & 120 \\
\hline 5 & No urgente & Azul & 240 \\
\hline
\end{tabular}

MTS: Manchester System Triage

El sistema se revisó en 2006 y se introdujeron las aportaciones recibidas en esos 10 años $^{29}$. A lo largo de este tiempo ha sido adoptado en muchos hospitales de todo el mundo ${ }^{30}$.

En España el Servicio de Urgencias del complejo Hospitalario de Ourense (Galicia), en el año 2002, realizó un estudio de validación y aplicación de MTS en nuestro medio, concluyendo que reúne las condiciones adecuadas para su aplicación ${ }^{31}$.

En el año 2003, se crea el Grupo Español de Triaje Manchester (GET-M) que se integra en el Grupo Europeo de Triaje Manchester el 30 de abril de 2004 y que participa en reuniones científicas del mismo.

\section{Análisis del MTS}

La escala de clasificación de pacientes contempla 52 motivos posibles de consulta que, de forma amplia se pueden agrupar en las 5 categorías siguientes: Enfermedad, Lesión, Niños, Conducta anormal e inusual y Catástrofes. En cada uno se despliega un árbol de flujo de preguntas. Después de 4-5 preguntas como máximo, clasifica al paciente en una de las 5 categorías que se traducen en un código de color y en un tiempo máximo de atención (Tabla 3).
El profesional selecciona un número de signos y síntomas de cada nivel de prioridad y utiliza discriminadores dispuestos en forma de diagramas para asignar a los pacientes a una de las 5 prioridades clínicas.

Los discriminadores generales son: riesgo vital, dolor, hemorragia, nivel de conciencia, temperatura y agudeza (tiempo de evolución) y se aplican a todos los pacientes independientemente de su forma de presentación.

El método es coherente en sus planteamientos ya que los síntomas guía pueden conducir a más de un diagrama de presentación clínica. Así, un paciente que no se encuentra bien con el cuello rígido y dolor de cabeza tendrá la misma prioridad si el profesional utiliza los diagramas de "adulto con mal estado general", "dolor de cuello" 0 "dolor de cabeza». En la figura 1 se representa un diagrama de flujo de los discriminadores generales y de uno de los 52 motivos de consulta.

Tampoco hace ninguna suposición sobre el diagnóstico y puede ser desarrollado por cualquier enfermero o enfermera independientemente de su grado de experiencia. Como requisito imprescindible se exige haber superado la formación inicial necesaria. 


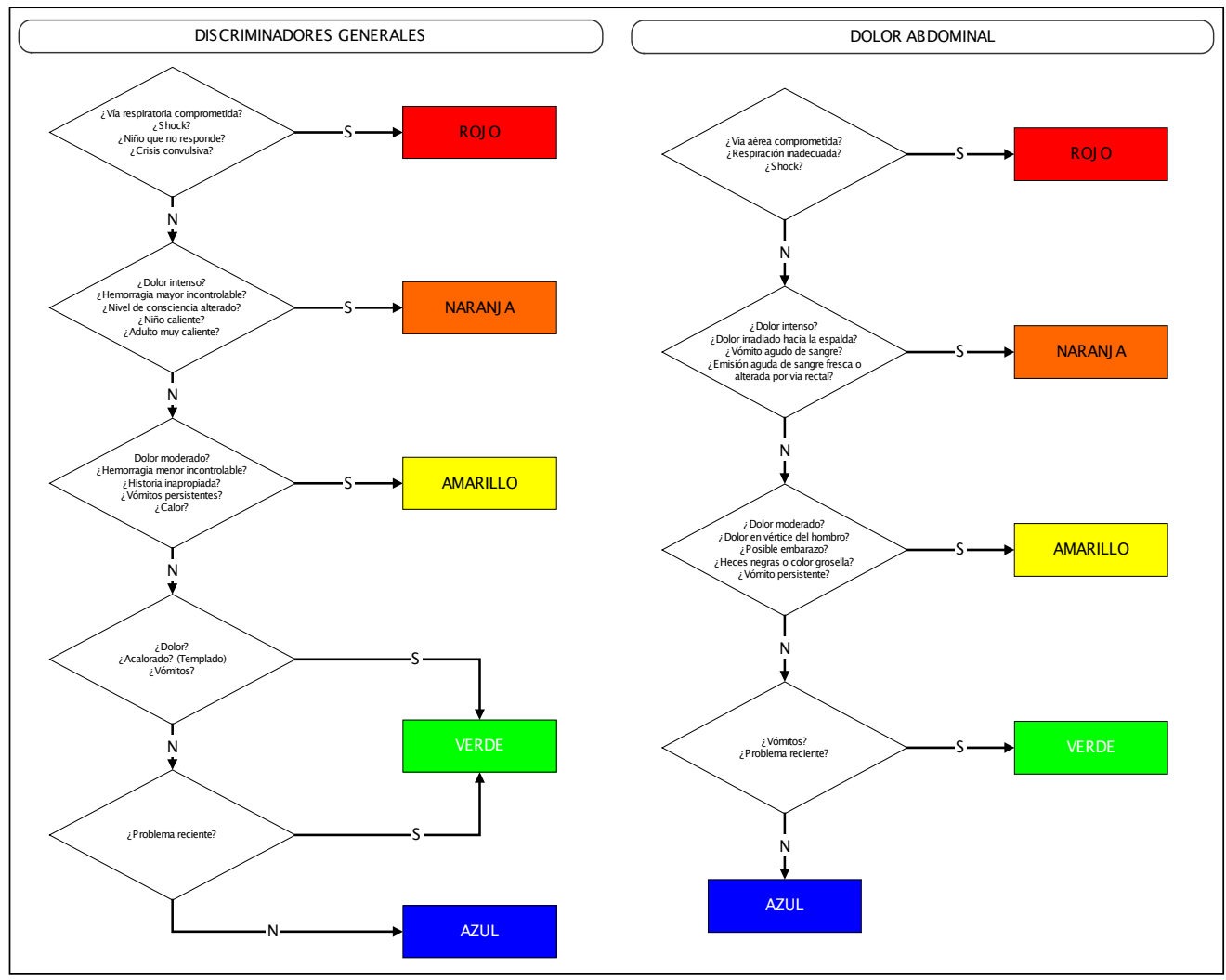

Figura 1. Diagrama de flujo de los discriminadores generales y del "dolor abdominal», uno de los 52 motivos de consulta del Sistema Manchester de Triaje.

El sistema tiene ya una experiencia acumulada de 14 años, permite realizar auditorias de calidad en la clasificación de pacientes tanto internas como externas, es válido, reproductible y aplicable, tiene una buena concordancia ${ }^{31}$ y es muy ágil y rápido en su aplicación cotidiana. Discrimina bien los paciente criticos $^{32,33} \mathrm{y}$ es aplicable tanto a pacientes adultos como a pacientes en edad pediátrica ${ }^{34}$.

El MTS es el sistema más ampliamente usado en hospitales de Europa y existen también grupos de triaje nacionales en países como Alemania, Holanda, Inglaterra e Irlanda ${ }^{35}$, Portugal y España. En el Reino Unido es el sistema mayoritariamente utilizado.

En España en la actualidad es el sistema elegido por los servicios de salud de Asturias, Galicia, Madrid y Comunidad Valenciana para sus hospitales públicos, 65 en total.

\section{SISTEMA DE TRIAJE HOSPITALARIO DE NAVARRA}

El 12 de mayo de 2008 se crea un grupo de trabajo con el objetivo de implantar un sistema de triaje estructurado de 5 niveles de priorización que aporte la mayor sensibilidad y fiabilidad que caracterizan a dichos sistemas ${ }^{36}$.

Se revisan otros modelos teóricos de triaje en vigor en otros ámbitos como son la NTS (2), la CTAS ${ }^{37-39}$, el SET $^{27}$ y el MTS $^{40}$ y, finalmente, se decide elaborar uno propio ya que obligatoriamente debe integrarse en el sistema informático que soporta la historia clínica informatizada ( $\mathrm{HCl}$ ) de la red pública de Navarra. Posteriormente se realizan cursos teórico-practico del nuevo modelo al personal de enfermería y médico. 
Este sistema integra los principios de los anteriormente analizados:

- Se aplica en base a 46 motivos de consulta (Tabla 4) con discriminantes, escalas de gravedad y pruebas complementarias.

Tabla 4. Motivos de consulta en el sistema de triaje hospitalario de Navarra.

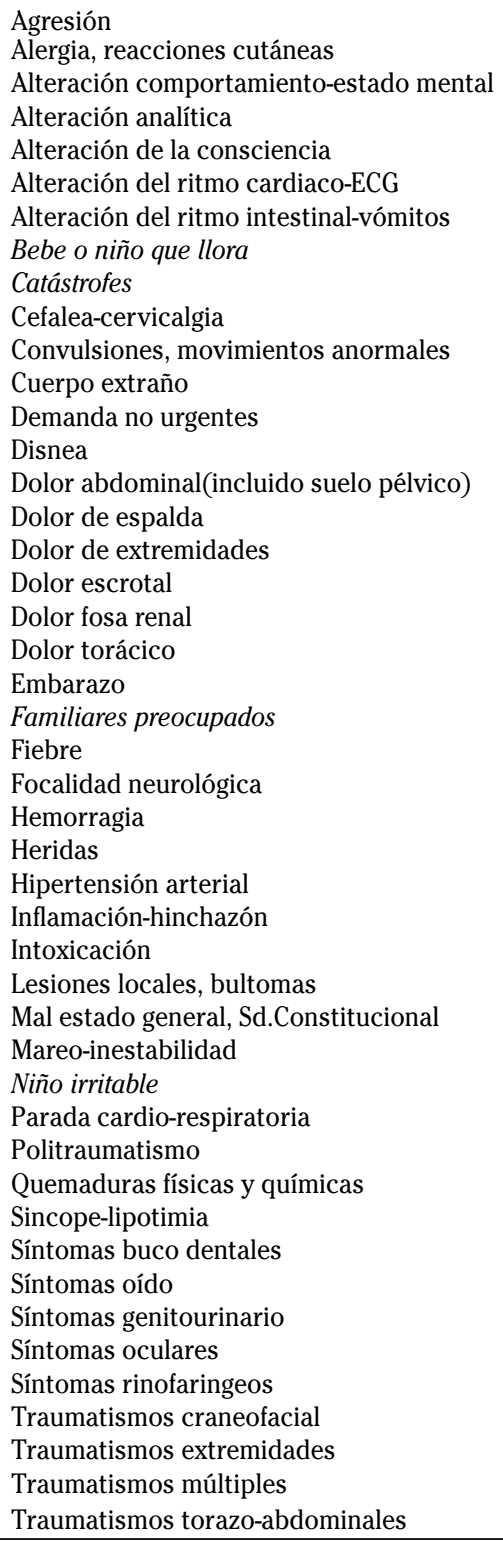

De ellos no se han desarrollado el de catástrofes ni los pediátricos.
- Se apoya en profesionales de enfermería y permite apoyo médico.

- Triaje de 5 niveles de priorización.

- Tiempos asistenciales para cada nivel de priorización.

- Incluye el concepto de re-triaje.

- Se integra en un sistema informático.

- Posibilita aplicar criterios de calidad y control al funcionamiento del propio servicio de urgencias.

- Permite su consulta desde otros puntos del sistema público sanitario, tanto hospitalarios como de atención primaria.

Cada nivel de priorización lleva asociado un tiempo máximo de atención médica y un color:

1. Nivel 1 (rojo)

a. Paciente de urgencia vital o con riesgo vital evidente

b. Tiempo de atención por médico y enfermera inmediato.

2. Nivel 2 (naranja)

a. Paciente emergente o con riesgo vital.

b. Tiempo de atención por enfermera inmediato y médico en $15 \mathrm{minu}-$ tos.

3. Nivel 3 (amarillo)

a. Paciente urgente.

b. Tiempo de atención por médico y enfermera en menos de 60 minutos.

4. Nivel 4 (verde)

a. Paciente menos urgente.

b. Tiempo de atención por médico y enfermera en 120 minutos.

5. Nivel 5 (azul)

a. Paciente no urgente.

b. Tiempo de atención por médico y enfermera en 240 minutos.

El re-triaje se debe realizar cuando el paciente ha superado estos tiempos de espera.

\section{Metodología del sistema}

Una vez realizada la entrevista (rápida, breve y dirigida) y elegido el motivo 
de consulta, disponemos de tres pantallas para desarrollar el triaje:

1. Pantalla general en la que aparece (Fig. 2):

- Anamnesis dirigida.

- Características de los síntomas y signos.

- Evaluación objetiva.

- Escalas de gravedad: que aparecen en determinados motivos de consulta.

- Escalas de dolor. Se aplica una escala de descripción verbal o descriptiva simple para el dolor ${ }^{41}$.

- Signos vitales ${ }^{42}$.

- Protocolos; dependiendo del ítem seleccionado nos solicita la realización de alguna prueba (glucemia, ECG....).
2. Pantalla con discriminadores (Fig. 3 ): discriminadores que se definen para cada motivo de consulta y habitualmente modifican el nivel de priorización. Aquí se incluyen también a los grupos especiales de pacientes que por sus características, morbimortalidad o vulnerabilidad requieren un abordaje especial en el área de urgencias, favoreciendo su nivel de prioridad ${ }^{43,44}$ : personas mayores de 80 años, embarazadas, enfermos crónicas y situaciones especiales (discapacitados, agredidos, tratamientos especiales, etc.).

3. Pantalla de constantes vitales y pruebas complementarias (Fig. 4): igual para todos los motivos de consulta. También pueden modificar el nivel de priorización.

Figura 2. Primera pantalla o pantalla general del sistema de triaje hospitalario de Navarra.

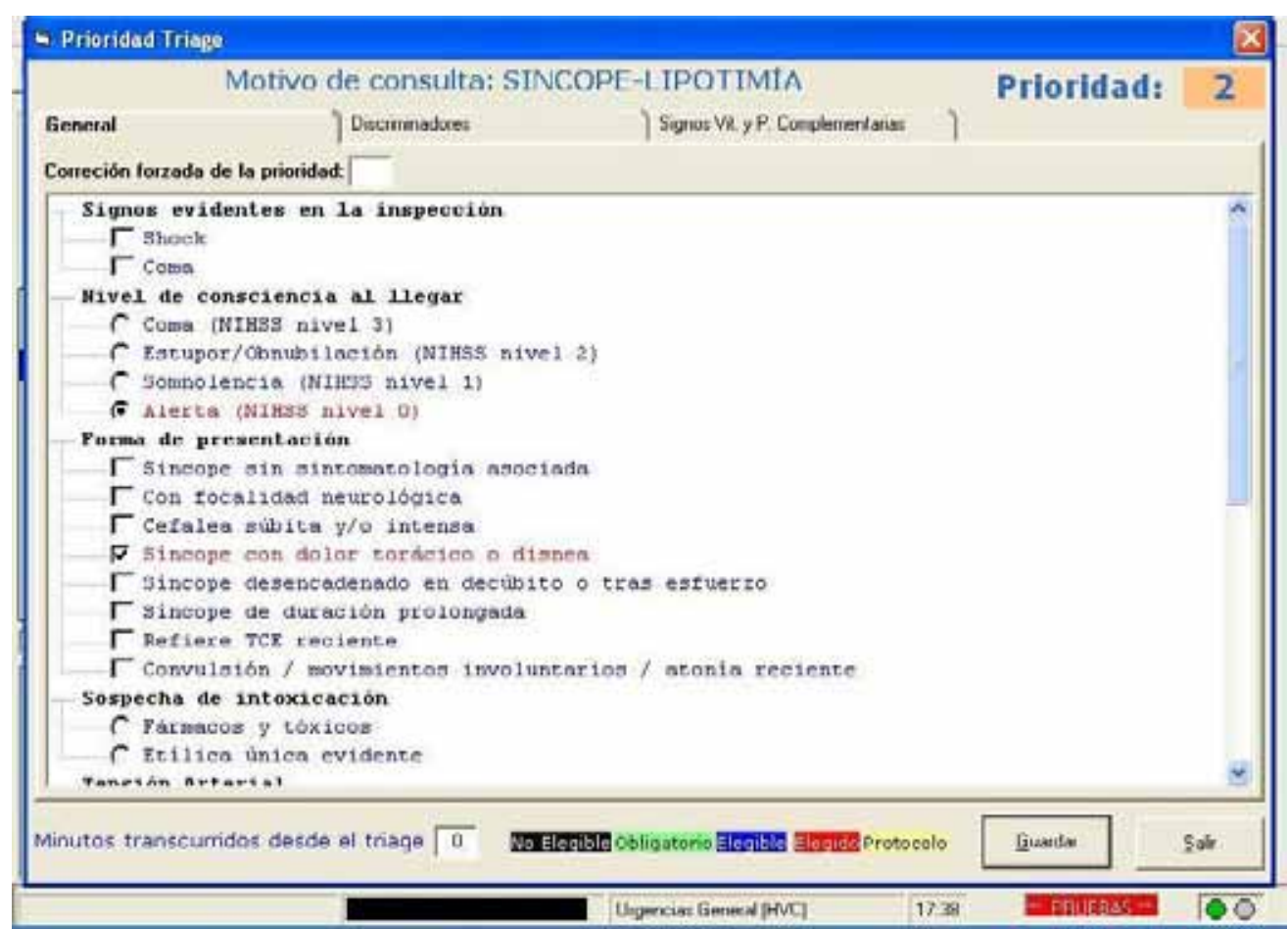




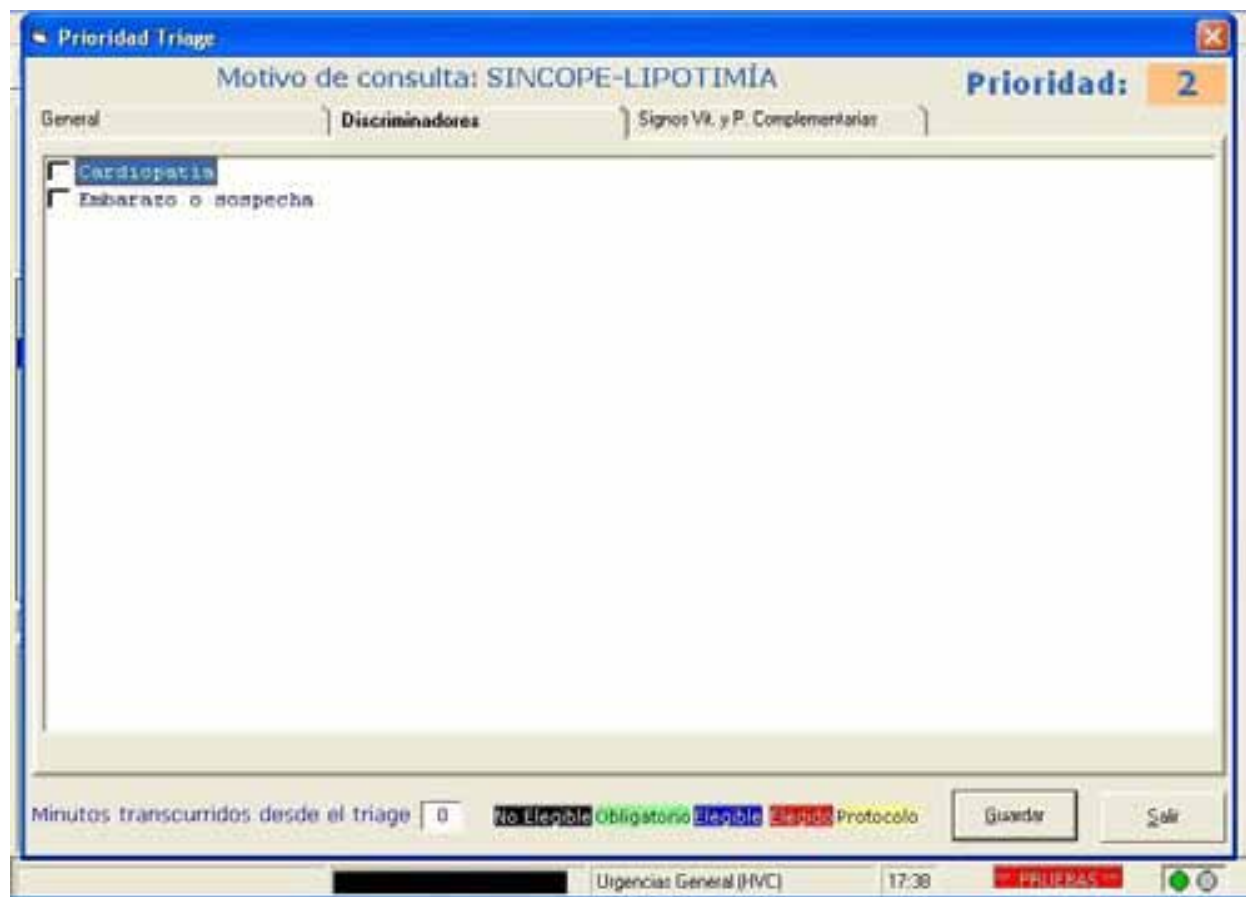

Figura 3. Segunda pantalla o pantalla de «discriminadores» del sistema de triaje hospitalario de Navarra.

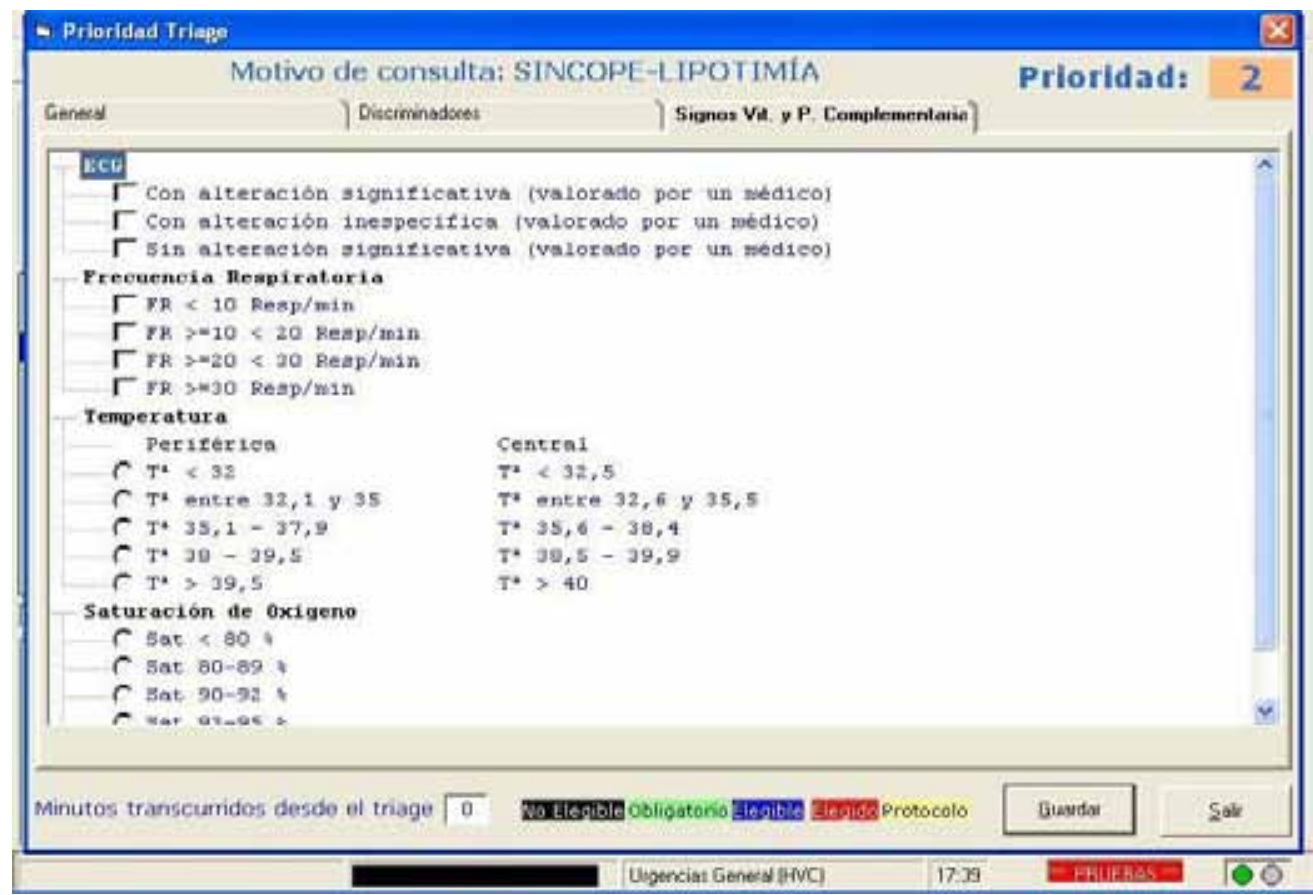

Figura 4. Tercera pantalla o pantalla de los signos vitales y pruebas complementarias del sistema de triaje hospitalario de Navarra. 
En la pantalla general se puede llevar a cabo una modificación "manual" del nivel de prioridad si el profesional estima que hay justificación no objetivable por el programa para hacerlo.

En todas las pantallas aparece el tiempo transcurrido desde que se efectúa el triaje, como testigo que permite controlar la necesidad de realizar un re-triaje. Para ello es necesario un sistema de aviso o de alarma de que ha transcurrido el tiempo límite.

Las ventajas más importantes de este sistema son:

1. Su implementación dentro del sistema informático sanitario de los hospitales públicos de Navarra y su complementación con el resto de fichas de la historia clínica informatizada.

2. Herramienta única y protocolos uniformes para todos los servicios de urgencias.

3. Accesible desde diferentes niveles asistenciales.

Tiene el inconveniente de su dependencia del resto de aplicaciones informáticas del sistema, por lo que no puede ser manejada como herramienta independiente.

En cuanto a sus posibilidades de monitorizar parámetros de calidad del propio servicio de urgencias, permite el análisis de todas aquellas variables relacionadas con los indicadores de tiempos, recursos, destinos, etc. En este sentido, tiene la ventaja de que el sistema informático que lo soporta puede acoplarse a sistemas de detección de pacientes por radiofrecuencia (RFID) por lo que sus posibilidades en cuanto a la precisión de toma de tiempos y localización de pacientes son máximas. En la actualidad, se va a iniciar un estudio piloto que permitirá valorar y validar este sistema.

\section{CONSIDERACIÓN FINAL}

El triaje se ha convertido en el sello de identidad del servicio de urgencias hospitalario que lo aplica, siendo además una herramienta objetiva que permite medir, evaluar y mejorar el funcionamiento y el rendimiento de dicho servicio.

Los actuales sistemas de triaje deben ser estructurados y basados en escalas de clasificación de cinco niveles. La asignación de dichos niveles debe fundamentarse en decisiones objetivas, apoyarse en algoritmos y sistemas informáticos que automaticen estas decisiones y permitir al mismo tiempo ajustes por parte del profesional que lo realiza.

Los profesionales de enfermería han demostrado capacidad para llevara cabo el triaje pero se valora positivamente la participación del médico en las decisiones relativas a los cuadros de mayor relevancia clínica.

El triaje debe extenderse a todos los ámbitos de la asistencia urgente y su desarrollo debe ir de la mano con el de los sistemas de información y con el de la tecnología.

\section{BIBLIOGRAFÍA}

1. Estadística de establecimientos sanitarios con régimen de internado, 2007. Instituto de Información sanitaria, Ministerio de Sanidad y Política Social, 2009.

2. Millá J. Urgencias médicas: algo más que una serie televisiva. Med Clin (Barc) 2001; 117: 295-296.

3. Zaragoza M, Calvo C, SaAd T, Morán FJ, San José S, Hernández P. Evolución de la frecuentación en un servicio de urgencias hospitalario. Emergencias 2009; 21: 339-345.

4. SÁnchez M, Salgado E, Miro O. Mecanismos organizativos de adaptación y supervivencia de lo servicios de urgencia. Emergencias 2008; 20: 48-53.

5. GómEz J. Urgencia, gravedad y complejidad: un constructo teórico de la urgencia basado en el triaje estructurado. Emergencias 2006; 18: 156-164.

6. Travers DA, Waller AE, Bowling JM, Flowers D, Tintinaldi J. Five-level triage more effective than three-level in tertiary emergency department. J Emerg Nurs 2002; 28: 395-400.

7. Wuerz RC, Fernández CM, Alarcón J. Inconsistency of Emergency department triage. Ann Emerg Med 1998; 32: 431-435. 
8. Wuerz RC, Travers D, Gilboy N, Eitel DR, RoSENAU A, YAZHARI R. Implementationand refinement of the Emergency Severity Index. Acad Emerg Med 2001; 8: 170-176.

9. Tudela P, Módol JM. Urgencias hospitalarias. Med Clin (Barc) 2003; 120: 711-716.

10. Gómez J. Clasificación de pacientes en los servicios de urgencias y emergencias: hacia un modelo de triaje estructurado de urgencias y emergencias. Emergencias 2003; 15: 165-174.

11. Mackway-Jones K, ed. Emergency Triage: Manchester Triage Group. London: BMJ Publishing Group, 1997.

12. Grupo de Triaje de la Sociedad Española de Medicina de Emergencias. Sistema Español de Triaje (SET). Madrid: Edicomplet 2004.

13. Gómez J, Faura J, Burgues L, Pàmies S. Gestión clínica de un servicio de urgencias hospitalario: indicadores de calidad, benchmarking y análisis de la casuística (case mix). Gestión hospitalaria 2004; 15: 3-12.

14. Beveridge R, Clarke B, Janes L. Canadian Emergency Department Triage and acuity scale implementation guidelines. CJEM 1999; 1 (Suppl 3): S1-S24.

15. Australasian College for Emergency Medicine. Policy Document-The Australasian Triage Scale. http://www.acem.org.au/open, documents/triage.htm (ed, 2000a)

16. Gómez Jiménez J, Puiguriguer J, Ferrando JB, et al. 1r Curs de formació en tria d'urgències. Bases conceptuals del Programa d’Ajuda al Triatge (PAT). Model Andorrà de Tria. Escaldes-Engordany, Desembre 2002-Abril 2003. http://www.colegidemetges.ad/sum/sum2. html

17. Gómez Jiménez J, Murray MJ, Beveridge R, Pons J, AlBert E, FERrANDo JB et al. Implementation of the Canadian Emergency Department Triage and Acuity Scale (CTAS) in the Principality of Andorra: Can triage parameters serve as EmergencyDepartment Quality Indicators? Can J Emerg Med 2003; 5: 315-322.

18. Peiró S, García-SEmpere A. El papel de los sistemas de clasificación de pacientes en la financiación de las urgencias hospitalarias. Gac Sanit 2003; 17: 441-443.

19. Gomez J, Segarra X, Prat J, Ferrando JB, Albert E, Borrás M. Concordancia, validez y utilidad del programa informático de ayuda al triaje (PAT) del Modelo andorrano de triaje (MAT). Emergencias 2003; 15: 339-344.

20. Sullivan R. Triage: a subspecialty of emergency nursing. Emphasis Nurs 1989; 3: 26-33.
21. Aranguren E, Capel JA, Solano M, Jean Louis C, LARumbe JC, ElEJALDE JI. Estudio de la validez pronóstica de la recepción, acogida y clasificación de pacientes en el área de urgencias en un hospital terciario. An Sist Sanit Navar 2005; 28: 177-188.

22. Morales JM, Martinez JD, Muñoz FJ. Consenso como método para la elaboración de estándares de recepción, clasificación e intervención sanitaria inicial de pacientes en urgencias: resultados y conclusiones (II). Tempus Vitalis 2003; 3: 15-24.

23. Subash F, Duna F, McNicholl B, Marlon J. Team Triage improves emergency department efficiency. Emerg Med J 2004; 21: 542-544.

24. Chan TC, KilleEn JP, Nelly D, Guss DA. Impact of rapad entry and accelerated carea t trage on reducing emergency department patien wait times, lengths of stay, and rate of left without being seen. Ann Emerg Med 2005; 46: 491-497.

25. Martín-SÁnchez FJ, González-Del Castillo J, Zamorano J, Candel FJ, González-Armengol JJ, Villarroel $\mathrm{P}$ et al. El facultativo, un elemento necesario para el triaje de un Servicio de Urgencias en un hospital terciario. Emergencias 2008; 20: 41-47.

26. Gómez J, Boneu F, Prat J, Becerra O, Albert E, Ferrando JB, Medina M. Validación clínica de la nueva versión del Programa de Ayuda al Triaje (web_e-PAT v3) del Modelo Andorrano de Triaje (MAT) y Sistema Español de Triaje (SET). Fiabilidad, utilidad y validez de la población pediátrica y adulta. Emergencias 2006; 18: 207-214.

27. Gómez Jiménez J, Torres Trillo M, López Pérez J, Jiménez Murillo L. Sistema Español de Triaje (SET). Madrid: Sociedad Española de Medicina de Urgencias y Emergencias (SEMES); 2004.

28. Gómez Jiménez J, Ferrando Garrigós JB, Vega García JL, Tomás Vecinas, Roqueta Egea F, Chanovas BorRÀs M. Model Andorrà de Triatge: Bases conceptuals i manual de formació. Principat d'Andorra: Ed. Gómez JiménezJ. Servei Andorrà d'Atenció Sanitària; 2004.

29. Emergency triage/Manchester triage Group; edited By Kevin Mackway-jones, Janet Marsden, Jill Windle. $2^{\text {nd }}$ ed. London BMJ Blackwell 1994.

30. Windle J. MackWAY-JONES K. Don't throw triage out with the bathwater. Emerg Med J 2003; 20: 119-120.

31. Triaje de urgencias hospitalarias editado por Kevin Mackway-Jones Editores de la version en castellano Blanco Ramos M, Caeiro Rey J, Pascual Clemente F. BMJ Publising, 2004. 
32. CooK MW, Jinks S. Does the Manchester triage system detect the critically ill? J Accid Emerg Med 1999; 16: 179-181.

33. Speake D, teece S, Mackway-Jones K. Detecting high-risk patients with chest pain. Emerg Nurse 2003; 11: 19-21.

34. Roukema J, steyerberg EW, Van Meurs A, Ruige M, VAN DER LeJ J, Moll HA. Validity of triage system in paediatric emergency care. Emerg Med J 2006; 23: 906-910.

35. CRoning JG. The Introduction of the Manchester Triage scale to an Emergency department in The Republic of Ireland. Accid Emerg Nurs 2003; 11: 121-125.

36. El sistema de 5 niveles de triage es más eficaz que de tres niveles en el servicio de urgencias terciario. (Five-level triage system more effective than three-level in tertiary emergency department) Emerg Nurs 2002; 28: 395-400.

37. Beveridge R. The Canadian Triage and acuity scale: A new and critical element in health care reform. J Emerg Med 1998; 16: 507-511.

38. MuRRAY MJ. The canadian triage and acuity scale: a canadian perspective on emergency department triage. Emerg Med 2003; 15; 6-10.
39. Michael M, Michael Bullard M, Graftein E. CTAS and CEDIS national working groups. Revisions to the Canadian emergency department triage and acuity scale Implementation Guidelines. Can J Emerg Med 2004; 6: 421-427.

40. Blanco MA, Caeiro JA, Pascual FA. Editores de la versión en castellano. Triaje de urgencias hospitalarios. Oxford: Blackwell Publishing ltd; 2004.

41. Serrano-Atero MS, Caballero J, Cañas A, García Saura PL, Serrano-Álvarez C. Pain assessment (I) Rev Soc Esp Dolor 2002: 9: 94-108.

42. Cooper RJ, Schringer DL, Fhaherty HL, Lin EJ HubBell KA. Effect of vital sings on triage decisions. Ann Emerg Med 2002; 39: 223-232.

43. Llorente S, Arcos M, Alonso M. Factores que influyen en la demora de un enfermo en un servicio de urgencias. Emergencias 2000; 12: 164-171.

44. Jiménez O, Conde A, Marchena J, Pavón JM, DÁVILA CD, BARBER P. Factores predictores del tiempo de estancia de los ancianos en un servicio de urgencias hospitalarias. Emergencias 2000; 12: 172-182. 